\title{
Fragile X Syndrome in a Female With Homozygous Full-Mutation Alleles of the FMR1 Gene
}

\author{
Farzane Vafaeie $^{1}$, Masoome Alerasool ${ }^{1,2}$, Nasrin Kaseb Mojaver ${ }^{1}$, Majid Mojarrad 1, 2, 3 \\ 1. Medical Genetics Laboratory, Genetic Foundation of Khorasan Razavi, Mashhad, IRN 2. Department of Medical \\ Genetics, Mashhad University of Medical Sciences, Mashhad, IRN 3. Genetic Research Center, Faculty of Medicine, \\ Mashhad University of Medical Sciences, Mashhad, IRN
}

Corresponding author: Majid Mojarrad, majidmojarrad12@gmail.com

\begin{abstract}
Fragile X syndrome (FXS) has been reported as the leading cause of mental retardation (MR) that predominantly involves males compared to females. An over-expansion of CGG repeats in the 5' untranslated region of the FMR1 gene plays the primary role in this disease. In this study, we encountered a homozygote female patient affected by FMR1 expansion mutation. Surprisingly, she had inherited her fullmutated alleles from two different ancestors. This condition is an extremely rare case of FXS. After accurate genetic counseling, family members were referred to the laboratory for genetic testing. Karyotype with two X chromosomes was the finding after the G-banding study of the proband.
\end{abstract}

Molecular analysis indicated that she was a female with full-mutated or pre-mutated alleles on both of her X chromosomes. It is a rare phenomenon that we detected in this patient. We have concluded that a combination of allele instability during oogenesis and inheritance of two alleles are the leading cause of MR in the presented case.

Categories: Genetics, Neurology, Pathology

Keywords: fragile x syndrome, fmr1 gene, cgg repeats, g-banding karyotype, mental retardation

\section{Introduction}

Fragile X syndrome (FXS, OMIM 300624), as the most common form of inherited neurodevelopmental disorder causing mental retardation (MR) and autism spectrum disorders, has been reported as the main cause of male MR in all races and ethnic groups. This X-linked dominant disease affects approximately 1:4000 males and 1:8000 females around the world. Meanwhile, MR and other clinical symptoms are considerably milder in affected female patients than males. The hemizygous feature of the $\mathrm{X}$ chromosome in males and a high density of the genes playing critical roles in central nervous system development on this chromosome could be reasonable explanations for this discrepancy [1]. An over-expansion of CGG repeats in the $5^{\prime}$ untranslated region (5'UTR) of the FMR1 gene, located on Xq27.3, is the primary genetic mechanism that explains the disease etiology. The expansion can be defined as four allelic forms, depending on the number of CGG repeats: normal allele with 6 to 40 repeats, intermediate allele with 41 to 60 repeats, premutation with 61 to 200 repeats, full-mutated allele with more than 200 repeats [2].

The 5' UTR CGG triplet repeats are the target site of DNA methylation in the mammalian genome. Therefore, full-mutated alleles impose a large amount of methylation in just the upstream region of FMR1 gene [3,4]. Due to the hyper-methylation condition and the following gene silencing, a dramatic reduction of FMR1 protein (FMRP) production occurs. FMRP is a critical protein for normal brain development, so FMRP loss or reduction leads to a wide range of autistic phenotypes $[5,6]$. Affected males suffer from moderate to severe MR and some specific behavioral patterns, playing as the main hallmarks after puberty. Intellectual disorder, puffiness around the eyes, soft skin, hypotelorism, prominent ears, long palpebral fissures, broad philtrum, facial hypotonia, macroorchidism, hyper-flexible joints, and autism-like behavior are some of the other main features of the patients $[1,7]$.

Studies have illustrated that, in contrast to the nature of dominant X-linked disease, only some heterozygous females with one full-mutated FMR1 allele show MR. At the same time, all the male patients suffer from a range of MR [8]. Intense skewed X chromosome inactivation in female patients could be the best explanation for this finding. In this regard, several studies have proved that the $\mathrm{X}$ inactivation ratio in FXS-affected patients skewed from 50\%:50\% toward 30\%:70\% with preference inactivation of the mutated X chromosome. Consequently, skewed X inactivation moderates the phenotype of fragile X in females [9]. Both males and females could complain from fragile-X-associated tremor/ataxia syndrome (FXTAS [MIM 300623]) as a direct consequence of FMR1 pre-mutation state; furthermore, premature ovarian failure (EXPOF [MIM 311360]) occurred in approximately $20 \%$ of females who carry a pre-mutated allele $[10,11]$. Pre-mutated allele expansion to full-mutated allele is identified in de novo cases of FXS. In several studies, the stretch of pre-mutated allele repeats has been confirmed during transmission from mothers to their 


\section{Cureus}

offspring. This transformation of the pre-mutations occurs through oogenesis and has rarely been reported during spermatogenesis [12]. However, females carrying full-mutated FMR1 might be suffering from mild autism-like behavior, anxiety, socio-emotional difficulties and learning problems, while homozygote females with two full-mutated alleles are supposed to be affected with fundamental aspects of the disease [9]. In this report, we present a female fragile X-affected patient with two full-mutated FMR1 genes that originated from two different ancestors.

\section{Case Presentation}

The patient was a 37-year-old female who had an intellectual disability with fertility problems (Figure 1 ). As shown in Figure 2, she was the first offspring of a consanguineous couple who manifested mild MR. The proband's sister had passed away of bone cancer at the age of 32. One of her paternal aunts and one of her paternal cousins were suffering from mild MR too. After the precise genetic counseling, family members were referred to the Genetic Foundation of Khorasan Razavi for genetic testing.

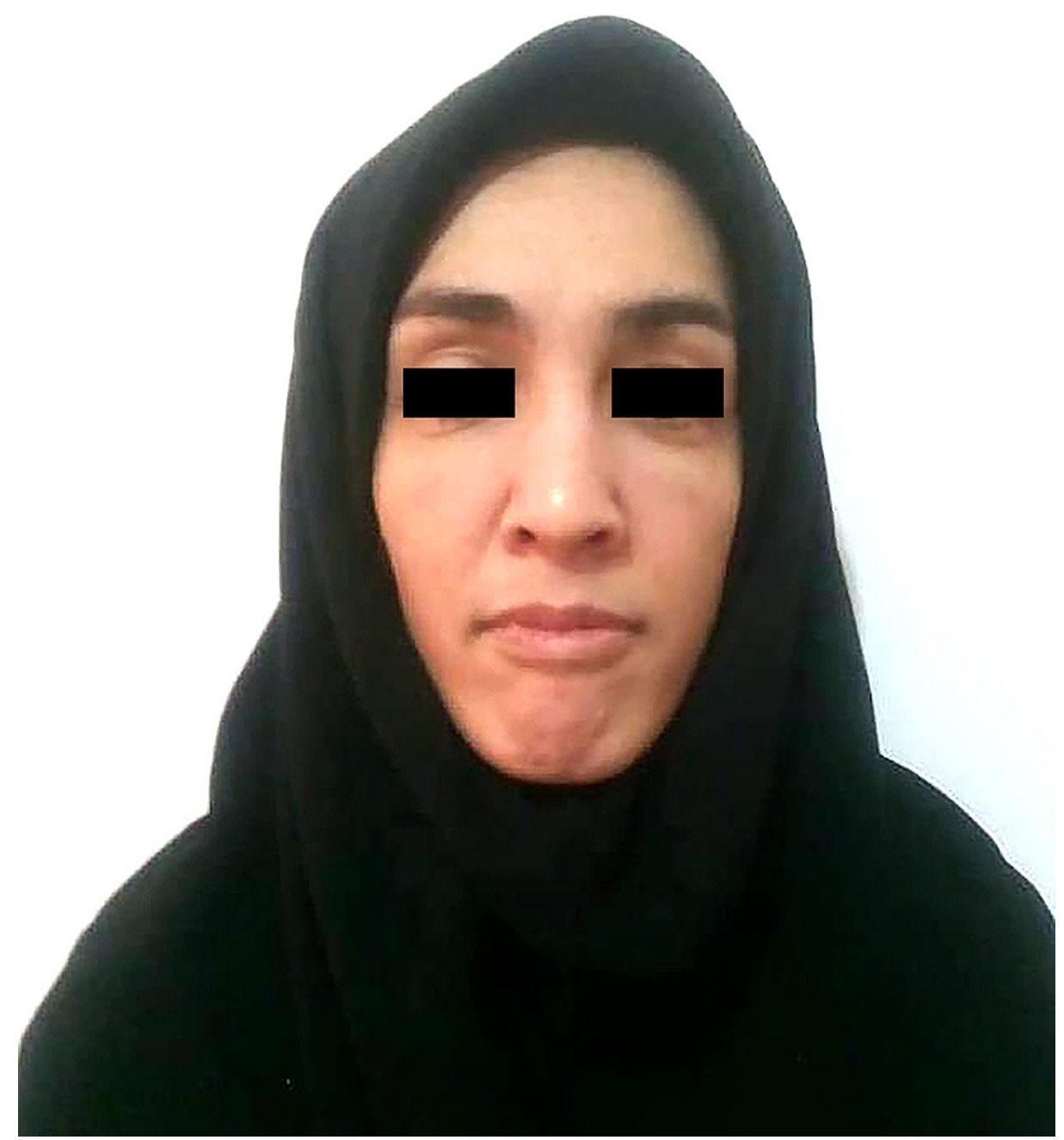

FIGURE 1: Image of the proband shows an elongated face due to a long forehead and a prominent chin 


\section{Cureus}

Familial mental retardation

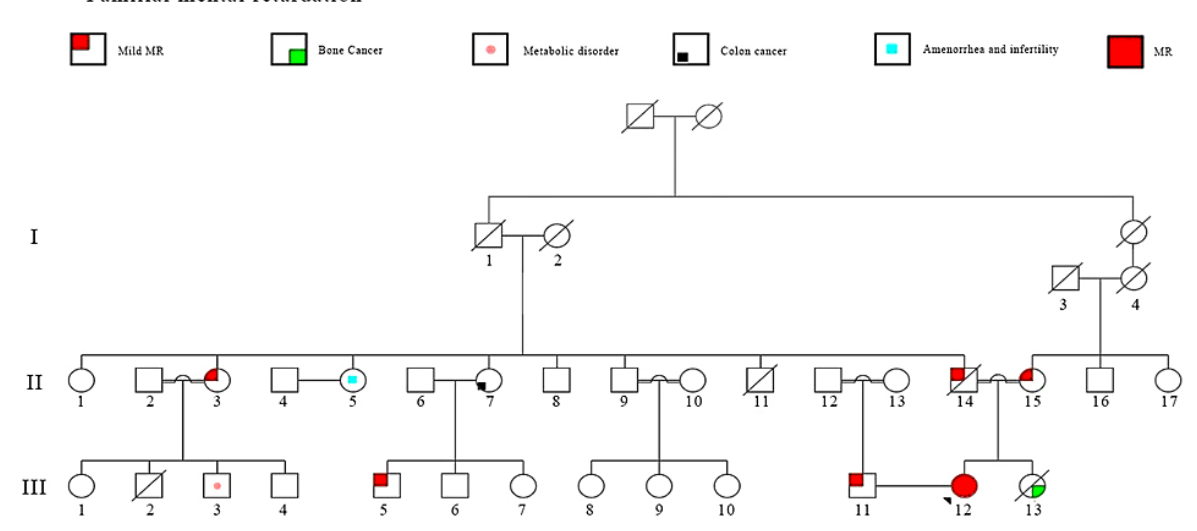

FIGURE 2: Family pedigree of the proband

The arrow indicates the proband (case III12) with MR phenotype. The parents (case II14, II15), paternal aunt (case II3) and one cousin (case III5) suffered from mild MR. Three consanguinities are seen in the family.

MR, mental retardation

According to the physical manifestation of proband's father, who had died a few years earlier, we suspected that he had been affected by FXS. Accordingly, the proband, her mother and other family members were examined for FXS using triplet-primed polymerase chain reaction (TP-PCR). As a brief, DNA extraction from peripheral blood samples was done using DNA isolation kit (Roche DNA Isolation Kit for Cells and Tissues, catalog no. 11814770001; Roche, Berlin, Germany). DNA qualification and quantification were examined using the Epoch Nanodrop UV-VIS Spectrophotometer (BioTek Instruments, VT, USA). Analysis of CGG repeats and detection of alleles with $>200$ repeats were done, and female zygosity was also resolved utilizing the TP-PCR method and FastFraX ${ }^{\text {TM }}$ FMR1 Sizing Kit (catalog no. F2-050-V; BioFactory Pte. Ltd., Singapore), followed by fragment analysis with the Applied Biosystems Genetic Analyzer (Thermo Fisher Scientific, MA, USA). Furthermore, TP-PCR was carried out on a paraffin-embedded tissue sample of the dead sister of the proband.

The patient and her husband were examined cytogenetically using the G-banding karyotype analysis method. Proband karyotype analysis using the G-banding technique indicated a female karyotype representing several spreads with one fragile $\mathrm{X}$ chromosome beside a normal copy of the $\mathrm{X}$ chromosome (Figure 3) and her husband revealed a normal karyotype. It is well known that G-banding karyotype analysis method is a complementary test and it cannot detect all fragile $\mathrm{X}$ chromosomes. 


\section{Cureus}

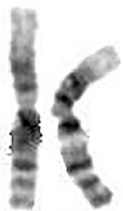

1

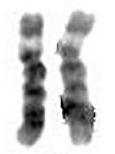

6

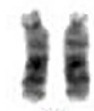

13

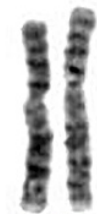

2

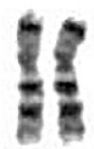

7

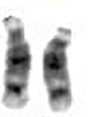

14

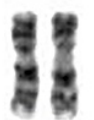

9

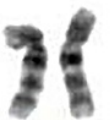

10

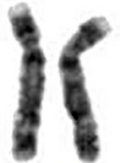

4

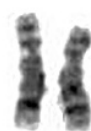

8

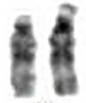

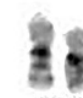

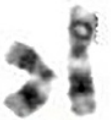

11
5
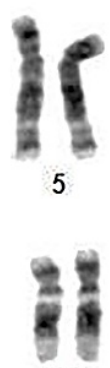

12

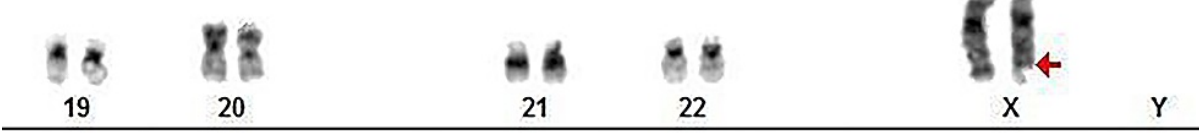

FIGURE 3: G-banding karyotype ( $\geq 500$ bands) showing apparently a normal $X$ and fragile $X[f r a(X)(q 27.3)]$ chromosomes (shown by a red arrow), in case III12 in the pedigree

Molecular analysis brightly showed two full-mutated alleles (>200 CGG) in the proband (case III12) (Figure $4 A$ ). The proband's mother (case II15) had a mild MR phenotype and was a heterozygous carrier of one premutated allele (Figure $4 B$ ). Analysis of extracted DNA from the paraffin-embedded tissue (case III13) detected a heterozygote of pre-mutation expansion (Figure 4C). Although the molecular analysis of the father (case II14), who had passed away, was impossible, based on the investigation, we realized that he also had been suffering from mild MR. Proband's paternal aunt (case II3), with one normal and one full-mutated allele resulting in a mild MR, had a normal daughter and three sons (Figure 4D). One of them passed away in early childhood because of infectious disease, the other suffers from metabolic disorder (hyperkalemic periodic paralysis), and the last son is normal. To examine the expansion of the repeats in some other family members, we analysed the expansion in three of the proband's cousins (III8, III9, and III10). All of them had the normal FMR1 alleles (Figures $4 E-4 G$ ). Table 1 shows the CGG repeats and mutation state in family members. 


\section{Cureus}

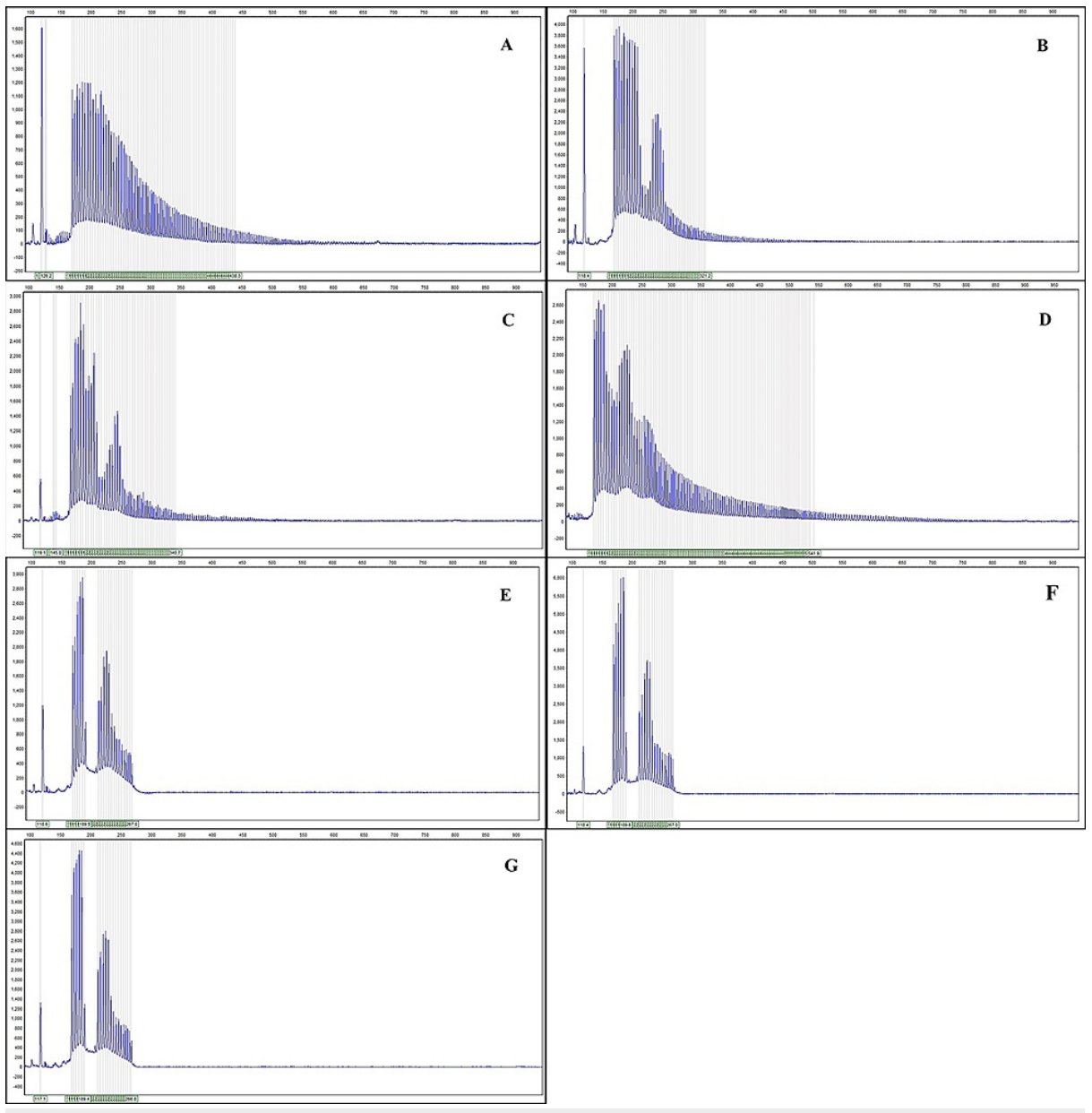

FIGURE 4: Graphs showing the number of CGG repeats in different members of the family: (A) case III12, (B) case II15, (C) case III13, (D) case II3, (E) case III8, (F) case III9, (G) case III10

\begin{tabular}{lll} 
Cases & CGG repeats & Mutation sta \\
\hline Case III12 & $>200$ & FM \\
\hline Case II15 & $136 / 25$ & PM carrier \\
Case III13 & $132 / 24$ & PM carrier \\
Case II13 & $245 / 29$ & FM carrier \\
Case III8 & $29 / 26$ & NL \\
Case III9 & $30 / 29$ & $\mathrm{NL}$ \\
Case III10 & $28 / 29$ & $\mathrm{NL}$
\end{tabular}

\section{TABLE 1: Results of diagnostic testing}

NL: normal alleles with 6-40 repeats; PM: pre-mutation with 61-200 repeats; FM: full mutation with more than 200 repeats.

With the analysis done in case III13, it could be concluded that the pre-mutated alleles are the unchanged pre-mutated alleles transferring from parents; in contrast, in the proband (case III12), the full-mutated alleles were the result of the repeat expansion through pre-mutated alleles' inheritance. 


\section{Discussion}

FXS can be diagnosed in both genders, while males are predominantly affected. Affected men by FXS mostly show MR and different ranges of autistic features and developmental delay, whereas females with nonspecific clinical features often have a less severe degree of the same disorders as in males. Owing to the presence of the second $\mathrm{X}$ chromosome with a healthy FMR1 gene in females, occasionally mild features are the only phenotypes that could be diagnosed in them even when they carry a pre-mutated or full-mutated FMR1 gene $[13,14]$. Recently Tabolacci et al. presented several FXS patients with fully methylated premutated alleles [15]. This epigenetic finding probably could be the main reason for the mild MR observed in females with the pre-mutated FMR1 gene. In our study, we have reported a family with females, who were carriers of either pre-mutated (case II15) or full-mutated FMR1 (case II3), and manifested mild MR. However, a controversial finding that has commonly been reported in many studies has described females with a full mutation in the FMR1 gene without remarkable MR. Various features of females with a fullmutated FMR1 gene are caused by extremely skewed X-chromosome inactivation [16]. In this study, according to the cytogenetic analysis of proband's chromosomes and triplet-primed PCR result, we have found that the proband had FXS with a full-mutated FMR1 gene in both of her X chromosomes. Some other studies have determined that some pre-mutated alleles (alleles with 61-200 repeats) are unstable and could be potential precursors of the full mutation and extend through transmission from the carrier mother to the offspring during oogenesis $[14,17]$. The full-mutated FMR1 alleles in the proband could be the result of the highly unstable pre-mutated FMR1 allele of her mother that is expanded through oogenesis; on the other hand, the proband's father had mild MR phenotype, and based on her paternal aunt (case II13) molecular analysis result, it can be hypothesized that the father could have carried a pre-mutation or full mutation on the FMR1 gene. Therefore, the proband as the offspring of the pre-mutated mother carrier and described father would carry a significant risk of being affected as a homozygous, carrying pre-mutation or full mutation $[18,19]$. Compound heterozygous female patients result from parents with two mutated alleles from a different origin. In comparison, female patients with homozygous expansion in the FMR1 gene are extremely rare. This problem often occurs due to the consanguineous marriage of an affected male with his carrier cousin; therefore, consanguinity and inbreeding raises the risk of $\mathrm{X}$-linked syndromes in females, as the situation increases the risk of common alleles in parents [20]. In 2020, Kim and colleagues described a three-year-old girl with typical FXS, with fully expanded FMR1 alleles (288 CGG repeats), resulting from uniparental isodisomy of the $\mathrm{X}$ chromosome transmitted from her mother. The latter carried a pre-mutated allele [14]. However, there are few reports on homozygous expansion in the FMR1 gene associated with FXS in females.

\section{Conclusions}

Due to the non-typical dysmorphic features and autism or autistic behavior in females affected with FXS, the diagnosis of FXS should be considered during genetic counseling of every female with a mental disability. Our case report indicates the importance of FMR1 gene analysis in females with intellectual disability or a family history of FXS. The possibility of being a carrier of FMR1 abnormal alleles for both parents should also be considered, especially if they are consanguineous or originated from a small and isolated community.

\section{Additional Information \\ Disclosures}

Human subjects: Consent was obtained or waived by all participants in this study. Conflicts of interest: In compliance with the ICMJE uniform disclosure form, all authors declare the following: Payment/services info: All authors have declared that no financial support was received from any organization for the submitted work. Financial relationships: All authors have declared that they have no financial relationships at present or within the previous three years with any organizations that might have an interest in the submitted work. Other relationships: All authors have declared that there are no other relationships or activities that could appear to have influenced the submitted work.

\section{Acknowledgements}

We are indebted to the family for their cooperation in performing this study. All participants signed a consent form. The Genetic Foundation of Khorasan Razavi supported this work.

\section{References}

1. Stembalska A, Łaczmańska I, Gil J, Pesz KA: Fragile X syndrome in females - a familial case report and review of the literature. Dev Period Med. 2016, 20:99-104.

2. Crawford DC, Acuña JM, Sherman SL: FMR1 and the fragile X syndrome: human genome epidemiology review. Genet Med. 2001, 3:359-371. 10.1097/00125817-200109000-00006

3. Fry M, Loeb LA: The fragile X syndrome $\mathrm{d}(\mathrm{CGG}) \mathrm{n}$ nucleotide repeats form a stable tetrahelical structure . Proc Natl Acad Sci U S A. 1994, 91:4950-4954. 10.1073/pnas.91.11.4950

4. Willemsen R, Levenga J, Oostra BA: CGG repeat in the FMR1 gene: size matters . Clin Genet. 2011, 80:214225. 10.1111/j.1399-0004.2011.01723.x

5. Bailey DB Jr, Hatton DD, Skinner M, Mesibov G: Autistic behavior, FMR1 protein, and developmental trajectories in young males with fragile X syndrome. J Autism Dev Disord. 2001, 31:165-174. 
6. Sherman S, Pletcher BA, Driscoll DA: Fragile X syndrome: diagnostic and carrier testing . Genet Med. 2005, 7:584-587. 10.1097/01.gim.0000182468.22666.dd

7. Bagni C, Tassone F, Neri G, Hagerman R: Fragile X syndrome: causes, diagnosis, mechanisms, and therapeutics. J Clin Invest. 2012, 122:4314-4322. 10.1172/JCI63141

8. Reiss AL, Freund L, Abrams MT, Boehm C, Kazazian H: Neurobehavioral effects of the fragile X premutation in adult women: a controlled study. Am J Hum Genet. 1993, 52:884-894.

9. Heine-Suñer D, Torres-Juan L, Morlà M, et al.: Fragile-X syndrome and skewed X-chromosome inactivation within a family: a female member with complete inactivation of the functional $\mathrm{X}$ chromosome. Am J Med Genet A. 2003, 122A:108-114. 10.1002/ajmg.a.20160

10. Hagerman RJ, Leavitt BR, Farzin F, et al.: Fragile-X-associated tremor/ataxia syndrome (FXTAS) in females with the FMR1 premutation. Am J Hum Genet. 2004, 74:1051-1056. 10.1086/420700

11. Rodriguez-Revenga L, Madrigal I, Alegret M, Santos M, Milà M: Evidence of depressive symptoms in fragileX syndrome premutated females. Psychiatr Genet. 2008, 18:153-155. 10.1097/YPG.0b013e3282f97e0b

12. Toledano-Alhadef H, Basel-Vanagaite L, Magal N, et al.: Fragile-X carrier screening and the prevalence of premutation and full-mutation carriers in Israel. Am J Hum Genet. 2001, 69:351-360. 10.1086/321974

13. Moreira de Mello JC, de Araújo ES, Stabellini R, et al.: Random X inactivation and extensive mosaicism in human placenta revealed by analysis of allele-specific gene expression along the $\mathrm{X}$ chromosome. PLoS One. 2010, 5:e10947. 10.1371/journal.pone.0010947

14. Kim JK, Jeong JE, Choi JM, Kim GH, Yoo HW: A female with typical fragile-X phenotype caused by maternal isodisomy of the entire X chromosome. J Hum Genet. 2020, 65:551-555. 10.1038/s10038-020-0735-9

15. Tabolacci E, Pomponi MG, Remondini L, et al.: Methylated premutation of the FMR1 gene in three sisters: correlating CGG expansion and epigenetic inactivation. Eur J Hum Genet. 2020, 28:567-575. 10.1038/s41431-019-0554-7

16. Chaste $\mathrm{P}$, Betancur $\mathrm{C}$, Gérard-Blanluet $\mathrm{M}$, et al.: High-functioning autism spectrum disorder and fragile $\mathrm{X}$ syndrome: report of two affected sisters. Mol Autism. 2012, 3:5. 10.1186/2040-2392-3-5

17. Hung CC, Lee CN, Wang YC, et al.: Fragile X syndrome carrier screening in pregnant women in Chinese Han population. Sci Rep. 2019, 9:15456. 10.1038/s41598-019-51726-4

18. Arlt MF, Durkin SG, Ragland RL, Glover TW: Common fragile sites as targets for chromosome rearrangements. DNA Repair (Amst). 2006, 5:1126-1135. 10.1016/j.dnarep.2006.05.010

19. Sun M, Ning J, Zhang H, Li S: Transmission of double FMR1 allelic premutations in a family . Genes Genom. 2017, 39:409-415. 10.1007/s13258-016-0506-3

20. Zlotogora J: Genetic disorders among Palestinian Arabs: 1. Effects of consanguinity . Am J Med Genet. 1997, 68:472-475. 10.1002/(sici)1096-8628(19970211)68:4<472::aid-ajmg20>3.0.co;2-o 